\title{
Impact De La Variabilité Pluviométrique Sur La Saison Culturale Dans La Zone De Production Cotonnière En Côte d'Ivoire
}

\author{
Charles Sékpa Dekoula, \\ Université Félix Houphouët-Boigny, Laboratoire de Sciences et Techniques \\ de l'Eau et de l'Environnement (LSTEE), Côte d'Ivoire

\section{Brou Kouame,} \\ Emmanuel Kouadio N'goran, \\ Fernand Guy Yao, \\ Centre National de Recherche Agronomique (CNRA) \\ Laboratoire Central Sols, Eaux et Plantes, Programme Gestion Durable des \\ Sols et Maîtrise de l'Eau, Programme coton, Côte d'Ivoire

\section{Jean-Nö̈l Ehounou, Nagnin Soro,} \\ Université Félix Houphouët-Boigny, Laboratoire de Sciences et Techniques \\ de l'Eau et de l'Environnement (LSTEE), Côte d'Ivoire
}

\begin{abstract}
In Côte d'Ivoire, agriculture is mostly rain-fed. As a result, changes and variations of climate have considerable impacts on crops production including cotton production. This paper focuses on analyzing the effects of rainfall variations on the cropping season (useful rain season) of cotton in cotton production area of Côte d'Ivoire. A set of stationarity tests was applied to the Nicholson index using rainfall data of the period 1950-2000. Then, from a frequency analyses, the variability of rainfall and characteristic parameters of the cropping season was evaluated in terms of risks. The result shows a general downward trend of rainfall in the cotton growing area with years of breaks between 1964 and 1975. Moreover, spatial evolution of the cropping season parameters is a function of latitude. After the years of breaks, the beginning and the end of the cotton cropping season, which became respectively later and earlier, indicated that the length of useful rain season became shorter. The deficits of seasonal rainfall accumulations vary up to $60 \%$. It is therefore necessary to update the crop calendar by taking into account variability of parameters of the useful rain season.
\end{abstract}


Keywords: Rainfall variability, cotton growing, cropping season, Côte d'Ivoire

\section{Résumé}

En Côte d'Ivoire, l'agriculture est en majorité de type pluvial. De ce fait, les changements et variations du climat ont des conséquences considérables sur la production des cultures, notamment celle du coton. C'est dans ce contexte que cette étude est menée afin d'analyser les effets des variations pluviométriques sur la saison culturale (saison des pluies utiles) du coton dans la zone cotonnière ivoirienne. Sur les données pluviométriques de la période 1950-2000, un ensemble de tests de stationnarité a été appliqué à l'indice de Nicholson. Puis, à partir d'une analyse fréquentielle, la variabilité des précipitations et des paramètres caractéristiques de la saison culturale a été évaluée en termes de risques. Les résultats montrent une tendance générale à la baisse dans la zone cotonnière avec des années de ruptures comprises entre 1964 et 1975. En outre, l'évolution spatiale des paramètres de la saison culturale est fonction de la latitude. Les dates de début et de fin de la saison culturale sont devenues respectivement plus tardives et précoces après la rupture et les longueurs quant à elles plus courtes. Les déficits des cumuls pluviométriques saisonniers varient jusqu'à $60 \%$. Il s'avère donc nécessaire d'actualiser le calendrier cultural tenant compte de la variabilité des paramètres de la saison des pluies utiles.

Mots-clés : Variabilité pluviométrique, culture du coton, saison culturale, Côte d'Ivoire

\section{Introduction}

À l'instar des autres pays d'Afrique en général et de l'Afrique de l'Ouest en particulier, la Côte d'Ivoire, pays à vocation agricole, n'est pas en marge des effets des changements climatiques. En effet, les travaux de Goula et al. (2006), Dongo et al. (2007) et Diomandé (2013) montrent que les modifications pluviométriques se font ressentir dans l'ensemble du pays. Selon Ahoussi et al. (2013), ces variabilités imprévisibles du climat ont considérablement perturbé les systèmes culturaux en Côte d'Ivoire. Ces fluctuations pluviométriques ont pour corollaire la variation des paramètres liés à la saison culturale (saison des pluies utiles) tels que : les dates de début et de fin, la durée et le cumul saisonnier. Il se pose alors le problème important de la détermination des dates de semis. Ces conditions nouvelles, qui se manifestent par une réduction de la productivité paysanne et une diminution des ressources financières sont vécues comme une contrainte majeure par les exploitants agricoles (Noufé et al., 2015). Cette dépendance de l'agriculture ivoirienne vis-à-vis du climat, notamment de la pluviométrie, met en avant la 
question de la vulnérabilité agricole et de l'insécurité alimentaire. Dès lors, cette situation devient une problématique de plus en plus récurrente pour les exploitants agricoles. Or, une connaissance de la saison des pluies utiles en termes de dates de début et de fin, de longueur et de cumul pluviométrique saisonnier pourrait aider les agriculteurs dans le choix des moments de semis et des variétés à semer pour minimiser les risques climatiques (Balme et al., 2005). L'objectif majeur de cet article est l'étude de l'effet de la variation de la pluviométrie sur la saison culturale du coton dans la zone cotonnière en Côte d'Ivoire.

\section{Matériels et méthodes \\ Site d'étude}

La zone de production de coton de la Côte d'Ivoire est située entre les longitudes $2^{\circ} 30$ et $8^{\circ} 30$ Ouest et les latitudes $6^{\circ} 50$ et $10^{\circ} 30$ Nord. Elle couvre une superficie d'environ $201693 \mathrm{~km}^{2}$ sur les $322462 \mathrm{~km}^{2}$ du pays (figure 1). Deux régimes pluviométriques caractérisent cette zone : le régime tropical de transition ou climat soudanais, caractérisé essentiellement par deux saisons bien marquées : la saison sèche en hiver boréal et la saison des pluies en été et le régime équatorial de transition atténué ou climat baouléen caractérisé par deux saisons des pluies et deux saisons sèches. La pluviométrie moyenne interannuelle varie entre 1055 et $1483 \mathrm{~mm}$. Trois sous-classes de sols ferralitiques sont distinguées dans cette zone : les sols sur roches basiques, les sols ferrugineux tropicaux et les sols hydromorphes (Koné, 2007).

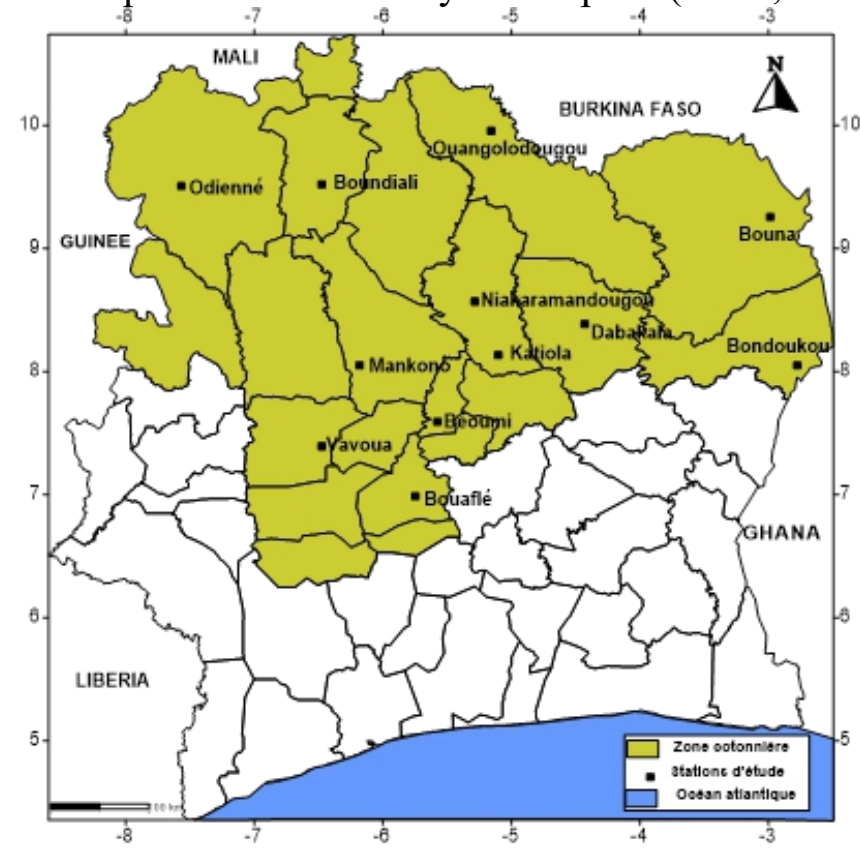

Figure 1 : Localisation de la zone d'étude en Côte d'Ivoire et stations pluviométriques associées 


\section{Matériel}

\section{Données pluviométriques}

Les données pluviométriques journalières de 1950 à 2000 ont été utilisées. Ces données proviennent de la Société d'Exploitation et de Développement Aéroportuaire, Aéronautique et Météorologique (SODEXAM) et du Laboratoire Central Sols, Eaux et Plantes (LCSEP) du Centre National de Recherche Agronomique (CNRA). Le choix de la période de cette série se justifie par l'arrêt des opérations de collecte de données dans les zones centre, nord et ouest pendant la crise militaro-politique de 2002 à 2010.

C'est après une critique des données pluviométriques que 12 stations (synoptiques et postes d'observations) ont été retenues. Ces stations répondent à une meilleure répartition spatiale de la zone cotonnière et offrent de longues séries d'observations pour les analyses. Pour cette étude, quatre localités assez représentatives des différentes zones climatiques ont été mises en évidence (tableau I).

Tableau I : Données pluviométriques des stations étudiées

\begin{tabular}{|c|c|c|c|c|}
\hline \multirow{2}{*}{ Stations } & \multirow{2}{*}{ Types de stations } & \multicolumn{2}{|c|}{ Coordonnées géographiques } & \multirow{2}{*}{ chroniques } \\
\cline { 3 - 4 } & & Longitudes & Latitudes & \\
\hline Béoumi (Centre) & Pluviométrique & $-5,57$ & 7,67 & $1950-2000$ \\
\hline Dabakala & Pluviométrique & $-4,43$ & 8,38 & $1950-2000$ \\
\hline Katiola & Pluviométrique & $-5,10$ & 8,13 & $1950-2000$ \\
\hline Bouaflé (Centre Ouest) & Pluviométrique & $-5,75$ & 6,98 & $1950-2000$ \\
\hline Vavoua & Pluviométrique & $-6,47$ & 7,37 & $1953-2000$ \\
\hline Bondoukou (Est) & Synoptique & $-2,78$ & 8,05 & $1950-2000$ \\
\hline Mankono (Centre & Pluviométrique & $-6,18$ & 8,05 & $1950-2000$ \\
\hline Nord) & & $-5,28$ & 8,67 & $1950-2000$ \\
\hline Niakara & Pluviométrique & $-6,47$ & 9,52 & $1950-2000$ \\
\hline Boundiali (Nord) & Pluviométrique & $-7,57$ & 9,50 & $1950-2000$ \\
\hline Odienné & Synoptique & $-5,15$ & 9,97 & $1950-2000$ \\
\hline Boungolo & Pluviométrique & $-3,60$ & 5,28 & $1950-2000$ \\
\hline
\end{tabular}

Stations de référence mises en évidence

\section{Méthodes}

Étude de la variabilité pluviométrique

\section{Indice pluviométrique de Nicholson centré réduit}

L'indice pluviométrique est le rapport de la différence entre la hauteur de précipitation annuelle à la station $i$ et la hauteur moyenne interannuelle de précipitation à l'écart type sur la période donnée. Les indices pluviométriques annuels sont calculés suivant la formule proposée par Nicholson et al. (1988) : $I_{i}=\frac{X_{i}-\bar{X}}{\sigma}$ 
Où $I_{i}:$ Indice pluviométrique de l'année i ;

$X_{i}$ : Pluviométrie annuelle de l'année i ;

$\bar{X}$ : Moyenne interannuelle de la pluviométrie sur la période étudiée ;

$\sigma$ : Valeur de l'écart type de la pluviométrie sur la période étudiée.

\section{Méthode de filtre passe-bas non récursif de Hanning d'ordre 2}

Une meilleure observation des fluctuations interannuelles s'obtient en éliminant les variations saisonnières. Dans ce cas, les totaux pluviométriques annuels sont pondérés en utilisant les équations suivantes recommandées par Assani (1999) :

$$
\begin{aligned}
& X_{(t)}=0,06 x_{(t-2)}+0,25 x_{(t-1)}+0,38 x_{(t)}+0,25 x_{(t+1)}+ \\
& 0,06 x_{(t+2)} \text { Pour } 3 \leq t \leq(n-2)
\end{aligned}
$$

Où : $X_{(t)}$ est le total pluviométrique pondéré du terme $t, x_{(t-2)}$ et $x_{(t-1)}$ sont les totaux pluviométriques des deux termes qui précèdent immédiatement le terme $t$, et $x_{(t+2)}$ et $x_{(t+1)}$ sont les totaux pluviométriques des deux termes qui suivent immédiatement le terme $t$.

Les totaux pluviométriques pondérés des deux premiers termes $\left(X_{(1)}\right.$, $\left.X_{(2)}\right)$ et des deux derniers termes $\left(X_{(n-1)}, X_{(n)}\right)$ de la série sont calculés au moyen des expressions suivantes ( $n$ étant la taille de la série) :

$X_{(1)}=0,54 x_{(1)}+0,46 x_{(2)}$

$X_{(2)}=0,25 x_{(1)}+0,50 x_{(2)}+0,25 x_{(3)}$

$\mathbf{X} \quad(\mathrm{n}-1) \quad=\mathbf{0 , 2 5} \quad(\mathrm{n}-2) \quad+\mathbf{0}, \quad 50 \mathrm{x} \quad(\mathrm{n}-1) \quad+\mathbf{0 , 2 5 x}$

$$
X_{(n)}=0,54 x_{(n)}+0,46 x_{(n-1)}
$$

Les grandes tendances sont également mises en évidence par une droite de régression de type affine : $\mathbf{y}=\mathbf{a x}+\mathbf{b}$; elle est obtenue par le calcul de la pente (a), qui est un coefficient directeur : $\mathrm{Si}$ a $>0$, on a une tendance à la hausse et si a $<0$, on a une tendance à la baisse.

\section{Détection de rupture dans les séries pluviométriques}

Le Programme de calcul "Khronostat», mis au point par HydroSciences Montpellier et disponible gratuitement sur le site SIEREM (http://www.hydrosciences.org/spip.php) (Boyer et al., 2006) a été utilisé pour détecter d'éventuelles ruptures dans les séries temporelles. Ce logiciel regroupe de nombreux tests spécifiques d'un changement de comportement de la variable dans la série temporelle. La détection de ruptures au sein des séries temporelles a nécessité l'application d'un ensemble de méthodes ; notamment le test de Pettitt (1979), la méthode de « U » Buishand (1984), la procédure bayésienne de Lee et Heghinian (1977) et la procédure de segmentation (Hubert et al. 1989). C'est à l'issue de l'application de ces différents tests, qu'une date de rupture détectée par la majorité des tests a été retenue. 


\section{Analyse des paramètres caractéristiques de la saison culturale ou saison des pluies utiles}

Le logiciel Instat + V.3.037 (Stern et al., 2006) a servi pour les analyses statistiques et agroclimatiques sur les paramètres clés de la saison culturale ou saison des pluies utiles [les dates (début et fin) et la longueur de la saison des pluies et le cumul pluviométrique saisonnier].

\section{Date de début et de fin de la saison des pluies utiles}

La détermination du début et de la fin de la saison des pluies utiles a été effectuée à partir de la méthode de Sivakumar (Sivakumar 1988 ; Balme et al., 2005 ; Stern et al., 2006) distinguée par son caractère agronomique. Cette méthode basée sur des seuils empiriques de précipitations a été reprise par Sarr (2007) et Goula et al. (2010) aux spécificités de la Côte d'Ivoire. Ainsi :

- Le début de la saison des pluies a été défini comme le premier jour, à partir du 15 mars (régime monomodal), lorsqu'on enregistre au moins $20 \mathrm{~mm}$ de pluie sur 2 jours successifs. Cependant, un épisode sec de plus de 07 jours consécutifs ne doit pas être observé pendant les 30 jours qui suivent le démarrage. En ce qui concerne le régime bimodal, le premier jour à partir du $1^{\text {er }}$ février a été retenu pour le démarrage de la grande saison et le $1^{\text {er }}$ août pour la petite saison des pluies tout en conservant les mêmes critères.

- La fin de saison des pluies, correspond au premier jour après une date fixée quand un sol capable de contenir $70 \mathrm{~mm}$ d'eau disponible est complètement épuisé par une perte journalière d'évapotranspiration de $4 \mathrm{~mm}$. Autrement dit, lorsque le bilan hydrique est nul. Pour le régime bimodal, cette date est fixée au $1^{\text {er }}$ juillet pour la grande saison des pluies et au $1^{\text {er }}$ octobre pour la petite saison. Quant au régime monomodal, cette date est fixée au $1^{\mathrm{er}}$ octobre.

\section{Longueur de la saison des pluies utiles}

La longueur de la saison est exprimée par la différence entre la date de fin et la date de début de la saison des pluies.

\section{Cumul pluviométrique saisonnier}

Le cumul pluviométrique saisonnier a été calculé en sommant les hauteurs de pluies tombées entre le début et la fin de la saison. Ce cumul pluviométrique obtenu a été comparé aux besoins totaux en eau du cotonnier.

Les analyses fréquentielles ont été réalisées pour les niveaux de probabilités de 20,50 et $80 \%$. Pour les paramètres de la saison des pluies utiles, ces probabilités 20,50 et $80 \%$ correspondent respectivement aux dates d'installation et de fin de saison précoce, normale et tardive des pluies utiles. $\mathrm{Au}$ niveau de la longueur de la saison, ces probabilités correspondent respectivement à des saisons courtes, normales et longues. Quant au cumul 
pluviométrique saisonnier, ces niveaux de probabilités correspondent respectivement à des années sèches, normales et humides (Sarr et al., 2011).

\section{Résultats}

\section{Variabilité interannuelle des précipitations}

\section{Tendance de la pluviométrie annuelle}

La représentation des indices centrés réduits de la pluviométrie moyenne interannuelle (1950-2000) dans les quatre stations assez représentatives de la zone cotonnière ivoirienne indique une tendance caractérisée par deux grandes périodes. Une période de hausse de la pluviométrie (période excédentaire) marquée par des valeurs des indices positifs et une période de baisse de la pluviométrie (période déficitaire) marquée par des valeurs d'indices négatifs. Aussi les courbes de tendance linéaire ont-elles toutes des pentes négatives $(\mathrm{a}<0)$, ce qui montre la baisse pluviométrique révélée par les séries de mesures.

À Boundiali, la période excédentaire s'étend de 1950 à 1975. Cependant une période de baisse pluviométrique par rapport à la tendance générale est observée entre 1958 et 1961. La période déficitaire quant à elle, s'étend entre 1976 et 2000. À Mankono, 1950-1964 marque la période excédentaire. La seconde période marquée par une tendance déficitaire, démarre à partir de 1965 avec une légère reprise à partir de 1998. À Béoumi, la période excédentaire se situe entre 1950 et 1964, tandis que la période déficitaire démarre en 1965. À Bouaflé, 1950-1972 marque la période excédentaire et 1973-2000 indique la période déficitaire (figure 2).

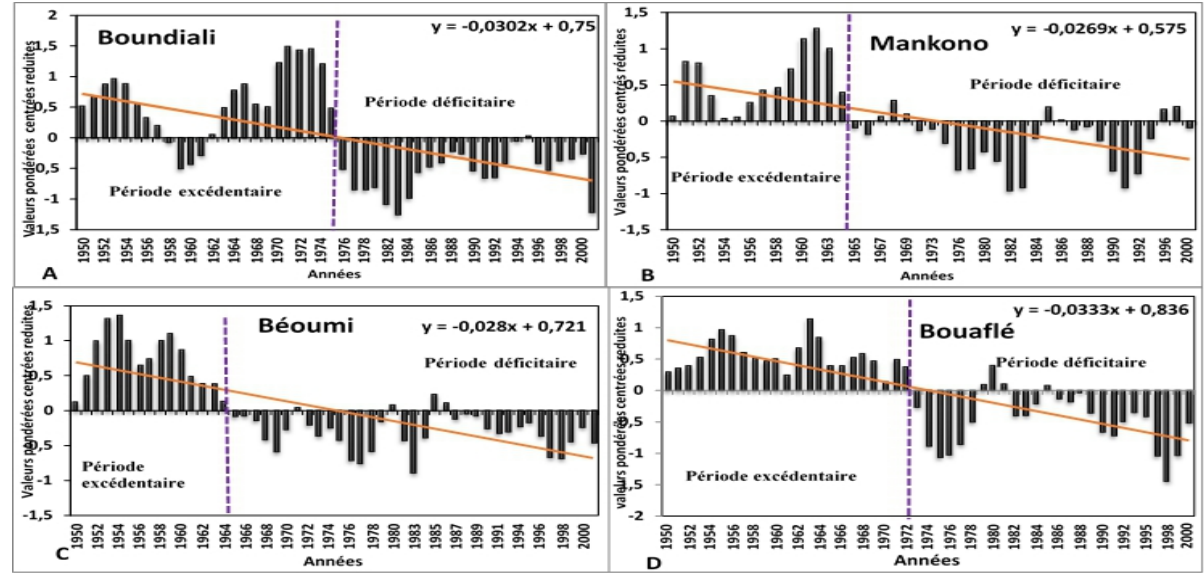

Figure 2 : Évolution interannuelle des indices pondérés centrés réduits de la pluviométrie moyenne pour quatre stations représentatives de la zone cotonnière de 1950 à 2000

\section{Détection des ruptures au sein des séries pluviométriques annuelles}

Les résultats des différents tests sont consignés dans le tableau II. 
L'analyse de ces résultats montre des dates de rupture spécifique à chaque station de référence de la zone d'étude. Ces dates de rupture détectées par la majorité des tests se situent entre 1964 et 1975. Elle est plus précoce à Béoumi et Mankono (1964) et plus tardive à Boundiali (1975). L'ensemble de ces résultats indique donc, un changement de moyenne dans la série pluviométrique des stations étudiées et une tendance générale à la baisse de la pluviométrie depuis la fin des années 1960 et le début des années 1970.

Tableau II : Ruptures des séries pluviométriques selon les différents tests

\begin{tabular}{cccccc}
\hline & \multicolumn{5}{c}{ Tests de rupture } \\
\cline { 2 - 6 } Station & Pettitt & Buishand & $\begin{array}{c}\text { Lee \& } \\
\text { Heghinian }\end{array}$ & $\begin{array}{c}\text { segmentation } \\
\text { de Hubert }\end{array}$ & $\begin{array}{c}\text { Date indiquée } \\
\text { par la majorité } \\
\text { des tests }\end{array}$ \\
\hline Béoumi & 1964 & 1964 & 1964 & 1963 & 1964 \\
Bouaflé & 1972 & 1972 & 1972 & 1972,1998 & 1972 \\
Mankono & 1964 & 1964 & 1969 & $1963 ; 1990$ & 1964 \\
Boundiali & 1975 & 1975 & 1975 & 1957,1961, & 1975 \\
\hline
\end{tabular}

Impact de la variabilité pluviométrique sur les paramètres de la saison des pluies utiles (saison culturale)

Date de début de la saison des pluies utiles

À Mankono (régime bimodal pas bien marqué) et Boundiali (régime monomodal), le démarrage normal $(50 \%)$ de la saison des pluies utiles s'effectue, entre le 22 et le 24 avril au cours de la période excédentaire (avant la rupture) et entre le 16 et 27 avril au cours de la période déficitaire (après la rupture). À Boundiali, les dates de démarrage précoces (20\%) et tardifs $(80 \%)$ sont retardées de 10 jours. Quant à Mankono, les dates de démarrage précoces et tardifs sont, respectivement avancées de 6 jours et retardées de 8 jours. À Béoumi et Bouaflé (régime bimodal) la grande saison des pluies, s'installe en moyenne entre le 16 et 24 mars avant la rupture et entre 27 et le 31 mars après la rupture. La variabilité des dates de début de la saison des pluies utiles dans ces localités est marquée par un retard compris entre 2 et 15 jours après la rupture. Quant au démarrage de la petite saison des pluies utiles, en années normales, elle est retardée de 8 jours à Béoumi et avancée de 4 jours à Bouaflé. Pour les démarrages précoces et tardifs, les dates varient peu après la rupture (Tableau III).

Tableau III : Variabilité des dates de début de la saison des pluies utiles pour quatre stations assez représentatives de la zone cotonnière avant et après la rupture.

\begin{tabular}{|c|c|c|c|c|}
\hline \multirow{2}{*}{ Stations } & \multirow{2}{*}{ Période } & \multicolumn{2}{|c|}{ Date de début de la saison des pluies } \\
\cline { 3 - 5 } & & $\mathbf{2 0} \%$ & $\mathbf{5 0} \%$ & $\mathbf{8 0} \%$ \\
\hline \multirow{5}{*}{ Boundiali } & \multicolumn{2}{|c|}{ Unique saison des pluies utiles } \\
\hline & $1950-1975$ & 6 avr. & 24 avr. & 15 mai \\
\cline { 2 - 5 } & $1976-2000$ & 16 avr. & 27 avr. & 25 mai \\
\cline { 2 - 5 } & Écart (jours) & 10 & 3 & 10 \\
\hline
\end{tabular}




\begin{tabular}{|c|c|c|c|c|}
\hline \multirow{3}{*}{ Mankono } & $1950-1964$ & 7 avr. & 22 avr. & 8 mai \\
\cline { 2 - 5 } & $1970-2000$ & 1 avr. & 16 avr. & 16 mai \\
\cline { 2 - 5 } & Écart (jours) & -6 & -6 & 8 \\
\hline \multirow{4}{*}{ Béoumi } & \multicolumn{6}{|c|}{ Grande saison des pluies utiles } \\
\cline { 2 - 5 } & $1950-1964$ & 9 mar. & 24 mar. & 12 avr \\
\cline { 2 - 5 } & $1968-2000$ & 15 mar. & 27 mar. & 16 avr. \\
\hline \multirow{3}{*}{ Bouaflé } & Écart (jours) & 6 & 3 & 4 \\
\cline { 2 - 5 } & $1950-1972$ & 8 mar. & 16 mar. & 11 avr. \\
\cline { 2 - 5 } & $1973-2000$ & 10 mar. & 31 mar. & 19 mar. \\
\cline { 2 - 5 } & Écart (jours) & 2 & 15 & 8 \\
\hline \multirow{3}{*}{ Béoumi } & $1950-1964$ & 4 août & 18 août & 29 août \\
\cline { 2 - 5 } & $1968-2000$ & 5 août & 26 août & 30 août \\
\cline { 2 - 5 } & Écart (jours) & 1 & 8 & 1 \\
\hline \multirow{3}{*}{ Bouaflé } & $1950-1972$ & 4 août & 26 août & 10 sept. \\
\cline { 2 - 5 } & $1973-2000$ & 3 août & 22 août & 12 sept. \\
\cline { 2 - 5 } & Écart (jours) & 1 & -4 & 2 \\
\hline
\end{tabular}

\section{Date de fin la saison des pluies utiles}

Dans l'ensemble, les dates de fin de la saison des pluies utiles est de plus en plus avancée après les années de rupture; et ce, quelle que soit la localité ou le régime pluviométrique. En ce qui concerne les localités à régime monomodal et bimodal moins marqués, la date de fin normale $(50 \%)$, est avancée de 8 jours à Boundiali et Mankono. Les dates de fins précoce (20\%) et tardive $(80 \%)$, sont respectivement avancées de 2 et 8 jour à Boundiali et de 7 et 12 jours à Mankono. Quant aux localités à régime bimodal (Béoumi et Bouaflé), les dates de fins (précoce, normal et tardive) de la grande saison des pluies utiles sont avancées d'au plus 9 jours après les dates de rupture. Cet avancement est compris entre 5 jours pour les dates précoces $(20 \%)$ et 25 jours pour les dates normales $(50 \%)$ pour la petite saison (Tableau IV).

Tableau IV : Variabilité des dates de fin de la saison des pluies utiles pour quatre stations assez représentatives de la zone cotonnière avant et après la rupture

\begin{tabular}{|c|c|c|c|c|}
\hline \multirow{2}{*}{ Stations } & Période & \multicolumn{3}{|c|}{ Date de fin de la saison des pluies utiles } \\
\cline { 3 - 5 } & \multicolumn{5}{|c|}{$\mathbf{2 0} \%$} & $\mathbf{5 0} \%$ & $\mathbf{8 0} \%$ \\
\hline \multirow{3}{*}{ Boundiali } & $1950-1975$ & 11 oct. & 29 oct. & 11 nov. \\
\cline { 2 - 5 } & $1976-2000$ & 9 oct. & 21 oct. & 3 nov. \\
\cline { 2 - 5 } & Écart (jours) & -2 & -8 & -8 \\
\hline \multirow{3}{*}{ Mankono } & $1950-1964$ & 12 oct. & 25 oct. & 9 nov. \\
\cline { 2 - 5 } & $1970-2000$ & 5 oct. & 17 oct. & 28 oct. \\
\cline { 2 - 5 } & Écart (jours) & -7 & -8 & -12 \\
\hline \multicolumn{7}{|c|}{ Grande saison des pluies utiles } \\
\hline \multirow{2}{*}{ Béoumi } & $1950-1964$ & 2 juil. & 9 juil & 19 juil. \\
\cline { 2 - 5 } & $1968-2000$ & 1 juil. & 2 juil. & 14 juil. \\
\hline
\end{tabular}




\begin{tabular}{|c|c|c|c|c|}
\hline & Ecart (jours) & -1 & -7 & -5 \\
\hline \multirow{3}{*}{ Bouaflé } & $1950-1972$ & 2 juil. & 10 juil. & 25 juil. \\
\cline { 2 - 5 } & $1973-2000$ & 1 juil. & 4 juil. & 16 juil. \\
\cline { 2 - 5 } & Écart (jours) & -1 & -6 & -9 \\
\hline \multirow{4}{*}{ Béoumi } & $1950-1964$ & 8 oct. & 21 oct. & 4 nov. \\
\cline { 2 - 5 } & $1968-2000$ & 3 oct. & 12 oct. & 28 oct. \\
\cline { 2 - 5 } & Écart (jours) & -5 & -9 & -7 \\
\hline \multirow{3}{*}{ Bouaflé } & $1950-1972$ & 21 oct. & 2 nov. & 14 nov. \\
\cline { 2 - 5 } & $1973-2000$ & 1 oct. & 8 oct. & 30 oct. \\
\cline { 2 - 5 } & Écart (jours) & -20 & -25 & -15 \\
\hline
\end{tabular}

\section{Longueur de la saison des pluies utiles}

Les localités étudiées sont marquées par une tendance au raccourcissement de la longueur de la saison des pluies après les années de rupture indiquées par la majorité des tests. Ce raccourcissement se traduit par des valeurs négatives des écarts. La durée normale $(50 \%)$ de la saison des pluies utiles est passée, de 189 à 175 jours à Boundiali, soit une diminution de 14 jours. A Mankono, elle est passée de 190 à 183 jours soit une différence de 7 jours. Les saisons de courtes durées $(20 \%)$ et longues durées $(80 \%)$ sont aussi réduites respectivement de 2 et 6 jours (Mankono) et de 2 et 12 jours (Boundiali). Dans la zone à régime bimodal, la grande saison des pluies de durée normale (50\%) est raccourcie de 10 et 12 jours après la rupture, respectivement à Béoumi et Bouaflé. Lorsque la grande saison des pluies utiles est de courte $(20 \%)$ ou longue $(80 \%)$ durée, elles sont respectivement raccourcies de 10 jours (Béoumi) et 14 jours (bouaflé) et de 1 jour (Bouaflé) et 10 jours (Béoumi). Quant à la petite saison des pluies utiles à Béoumi, les longueurs normales, courtes et longues sont raccourcies respectivement de 7 jours, 12 jours et 8 jours. A Bouaflé, les longueurs, normales, courtes et longues sont raccourcies respectivement de 15 jours, 25 jours et 16 jours. (Tableau V).

Tableau V : Variabilité de la longueur de la saison des pluies utiles pour quatre stations assez représentatives de la zone cotonnière avant et après la rupture.

\begin{tabular}{|c|c|c|c|c|}
\hline \multirow{2}{*}{ Stations } & \multirow{2}{*}{ Période } & \multicolumn{3}{|c|}{ Longueur de la saison des pluies } \\
\cline { 3 - 5 } & & $20 \%$ & $50 \%$ & $80 \%$ \\
\hline \multirow{3}{*}{ Boundiali } & $1950-1975$ & 155 & 189 & 210 \\
\cline { 2 - 5 } & $1976-2000$ & 153 & 175 & 198 \\
\cline { 2 - 5 } & Écart (jours) & -2 & -14 & -12 \\
\hline \multirow{5}{*}{ Mankono } & $1950-1964$ & 162 & 190 & 204 \\
\cline { 2 - 5 } & $1970-2000$ & 156 & 183 & 202 \\
\cline { 2 - 5 } & Écart (jours) & -6 & -7 & -2 \\
\hline
\end{tabular}




\begin{tabular}{|c|c|c|c|c|}
\hline \multirow{3}{*}{ Béoumi } & $1950-1964$ & 87 & 111 & 123 \\
\cline { 2 - 5 } & $1968-2000$ & 77 & 101 & 113 \\
\cline { 2 - 5 } & Écart (jours) & -10 & -10 & -10 \\
\hline \multirow{3}{*}{ Bouaflé } & $1950-1972$ & 94 & 111 & 131 \\
\cline { 2 - 5 } & $1973-2000$ & 80 & 99 & 130 \\
\cline { 2 - 5 } & Écart (jours) & -14 & -12 & -1 \\
\hline \multirow{5}{*}{ Péoumi } & $1950-1964$ & 46 & 63 & 81 \\
\cline { 2 - 5 } & $1968-2000$ & 34 & 56 & 73 \\
\cline { 2 - 5 } & Écart (jours) & -12 & -7 & -8 \\
\hline \multirow{2}{*}{ Bouaflé } & $1950-1972$ & 50 & 69 & 99 \\
\cline { 2 - 5 } & $1973-2000$ & 25 & 54 & -16 \\
\cline { 2 - 5 } & Écart (jours) & -25 & -15 & 83 \\
\hline
\end{tabular}

\section{Cumul pluviométrique saisonnier}

Dans les zones à régime monomodal et bimodal moins marqué, le cumul pluviométrique saisonnier en années normales (50\%), est passé de $1604 \mathrm{~mm}$ à $1314 \mathrm{~mm}$ après la rupture, soit un déficit de $18 \%$ pour la localité de Boundiali. En années sèches $(20 \%)$ et humide $(80 \%)$, ce cumul pluviométrique saisonnier est passé respectivement de $1257 \mathrm{~mm}$ à $1126 \mathrm{~mm}$ soit un déficit de $10 \%$ et de $1863 \mathrm{~mm}$ à $1648 \mathrm{~mm}$ soit un déficit de $12 \%$. Quant à la localité de Mankono, en années normales, ce cumul pluviométrique saisonnier est passé de $1036 \mathrm{~mm}$ à $891 \mathrm{~mm}$ soit un déficit de $14 \%$. En années sèches et humides, ce cumul pluviométrique saisonnier est passé respectivement de $741 \mathrm{~mm}$ à $742 \mathrm{~mm}$ soit un déficit de moins de $1 \%$ et de $1261 \mathrm{~mm}$ à $1114 \mathrm{~mm}$ soit un déficit de $12 \%$. Le cumul pluviométrique saisonnier de la grande saison des pluies, en années normales est passé de 477 $\mathrm{mm}$ à $427 \mathrm{~mm}$ soit un déficit de $10 \%$ à Béoumi. A Bouaflé ce cumul est passé de $639 \mathrm{~mm}$ à $499 \mathrm{~mm}$ soit un déficit de $22 \%$. En années sèches et humides les cumuls saisonniers sont passés respectivement de $357 \mathrm{~mm}$ à $339 \mathrm{~mm}(5 \%$ de déficit) et de $655 \mathrm{~mm}$ à $542 \mathrm{~mm}$ (17\% de déficit) à Béoumi. Dans la localité de Bouaflé ces mêmes cumuls saisonniers sont passés respectivement de 462 $\mathrm{mm}$ à $357 \mathrm{~mm}$ ( $23 \%$ de déficit) et de $760 \mathrm{~mm}$ à $676 \mathrm{~mm}$ (11\% de déficit). Quant à la petite saison des pluies, le cumul pluviométrique saisonnier, en années normales, est passé de $390 \mathrm{~mm}$ à $339 \mathrm{~mm}$ (13\% de déficit) et de 451 $\mathrm{mm}$ à $302 \mathrm{~mm}$ (33\% de déficit) respectivement à Béoumi et Bouaflé. Les cumuls pluviométriques saisonniers, en années sèches et humides, sont passés respectivement de $255 \mathrm{~mm}$ à $186 \mathrm{~mm}$ ( $27 \%$ de déficit $)$ et de $564 \mathrm{~mm}$ à 380 mm (32\% de déficit) à Béoumi. Dans la localité de Bouaflé, ils sont passés respectivement de $343 \mathrm{~mm}$ à $138 \mathrm{~mm}(60 \%$ de déficit $)$ et de $630 \mathrm{~mm}$ à 513 mm (19\% de déficit) (Tableau VI). 
Tableau VI : Variabilité du cumul pluviométrique saisonnier pour les quatre stations représentatives de la zone cotonnière avant et après la rupture

\begin{tabular}{|c|c|c|c|c|}
\hline \multirow{2}{*}{ Stations } & \multirow[t]{2}{*}{ Période } & \multicolumn{3}{|c|}{ Cumul saisonnier (mm) } \\
\hline & & $20 \%$ & $50 \%$ & $80 \%$ \\
\hline \multicolumn{5}{|c|}{ Unique saison des pluies } \\
\hline \multirow[t]{3}{*}{ Boundiali } & $1950-1975$ & 1257 & 1604 & 1863 \\
\hline & $1976-2000$ & 1126 & 1314 & 1648 \\
\hline & Déficit (\%) & -10.42 & -18.08 & -11.54 \\
\hline \multirow[t]{3}{*}{ Mankono } & 1950-1964 & 741 & 1036 & 1261 \\
\hline & $1970-2000$ & 742 & 891 & 1114 \\
\hline & Déficit (\%) & 0,13 & -14 & $-11,66$ \\
\hline \multicolumn{5}{|c|}{ Grande saison des pluies } \\
\hline \multirow[t]{3}{*}{ Béoumi } & $1950-1967$ & 357 & 477 & 655 \\
\hline & $1968-2000$ & 339 & 427 & 542 \\
\hline & Déficit (\%) & $-5,04$ & $-10,48$ & $-17,25$ \\
\hline \multirow[t]{3}{*}{ Bouaflé } & 1950-1972 & 462 & 639 & 760 \\
\hline & $1973-2000$ & 357 & 499 & 676 \\
\hline & Déficit (\%) & $-22,73$ & $-21,91$ & $-11,05$ \\
\hline \multicolumn{5}{|c|}{ Petite saison des pluies } \\
\hline \multirow[t]{3}{*}{ Béoumi } & $1950-1967$ & 255 & 390 & 564 \\
\hline & $1968-2000$ & 186 & 339 & 380 \\
\hline & Déficit (\%) & $-27,06$ & $-13,08$ & $-32,62$ \\
\hline \multirow[t]{3}{*}{ Bouaflé } & 1950-1972 & 343 & 451 & 630 \\
\hline & $1973-2000$ & 138 & 302 & 513 \\
\hline & Déficit (\%) & $-59,77$ & $-33,04$ & $-18,57$ \\
\hline
\end{tabular}

\section{Discussion}

La saison des pluies utiles ou saison culturale de la quelle dépend la période d'activités agricoles est, depuis quelques décennies, confrontée à des perturbations. En effet, l'examen des graphes des indices pluviométriques et des courbes de tendances, indique des périodes de hausse et de baisse puis une tendance générale à la baisse des indices. Ces résultats montrent que la zone étudiée a subi une récession pluviométrique. Si la déforestation ne peut pas à elle seule être la principale responsable de cette baisse de la pluviométrie, elle y a certainement contribué. Cette récession pluviométrique a été révélée par les travaux de Paturel et al. (1995) en Côte d'Ivoire. Fossou et al. (2015) ont également rapporté les mêmes résultats dans le Centre-Est de la Côte d'Ivoire.

Les dates de rupture obtenues pour les séries pluviométriques se situent entre 1964 et 1975. Ces années de rupture coïncident avec le découpage donné par le filtre passe-bas de Hanning d'ordre 2. Ces dates de rupture sont proches de la période de rupture (1966-1971) de la majorité des stations pluviométriques identifiée en Côte d'Ivoire par Paturel et al. (1997) et Servat et al. (1999). Ces résultats sont également en accord avec ceux de Mahé et Olivry (1995) puis Paturel et al. (1997). Leurs travaux ont montré que les 
dates de rupture ont subi des fluctuations entre la fin des années 1960 et le début des années 1970 en Afrique de l'Ouest et Centrale. Toutefois, selon Noufé (2011), si la majorité des auteurs s'accorde à reconnaître que la rupture s'est globalement produite en Côte d'Ivoire autour de 1970, ce n'est qu'à titre indicatif; et pour preuve les dates de décrochage trouvée ici, sont soit antérieur (1964), soit postérieur (1975) à cette date.

L'analyse des cumuls pluviométriques saisonniers a montré qu'en années sèches $(20 \%)$, normale $(50 \%)$ et humides $(80 \%)$, après la rupture, ces cumuls ont enregistré des déficits allant jusqu'à $60 \%$. Selon Lebel et Ali (2009), ces changements de cumul saisonnier sont la conséquence d'une diminution du nombre d'évènement pluvieux et non d'une baisse d'intensité des pluies. Goula et al. (2006), indiquent également que, les principaux facteurs responsables de ces anomalies pluviométriques sont à l'échelle régionale ou globale. Cependant, en prenant en compte les quantités de pluies saisonnières après rupture, on retient que la zone cotonnière est potentiellement apte à satisfaire les exigences hydriques du cotonnier. Selon Cretenet et al., (2002), les besoins en eau du cotonnier sont estimés à $500 \mathrm{~mm}$ pour son cycle. Toutefois, cette plante nécessite une bonne répartition des quantités de pluies durant les différentes phases phénologiques qui sont des périodes critiques.

La connaissance des paramètres tels que les dates de début et de fin de la saison des pluies utiles et sa longueur, offre un intérêt pour la planification des activités agricoles. Cependant, l'évolution de ces paramètres indique une variation spatio-temporelle dans la zone cotonnière. Du point de vue spatial, la saison des pluies utiles (saisons unique et grande saison) s'installe de manière progressive du Centre-Ouest (Bouaflé) au Nord (Boundiali) en passant par le Centre (Béoumi) et le Centre-Nord (Makono). Cette évolution montre que la date de début de la saison varie en fonction de la latitude. Aussi, traduit-elle la progression de la mousson du Sud vers le Nord. Cette évolution latitudinale a été mise en évidence par Noufé et al. (2015) dans l'Est ivoirien. En revanche, la fin de la saison des pluies utiles ne suit pas le même gradient et varie d'une zone à une autre.

La comparaison des paramètres clés de la saison, avant et après la rupture, indique une variation temporelle. L'on note en moyenne, des débuts de saison des pluies de plus en plus tardifs après les années de ruptures. Ces résultats confirment ceux de Goula et al. (2010), qui ont noté qu'en Côte d'Ivoire, le début des saisons des pluies accuse un retard de 1 à 10 jours presque dans l'ensemble du pays. Cependant les localités comme Bouna dans le Nord-Est enregistrent un retard de plus de 10 jours dans l'installation de la saison des pluies. Agbangla (1998) a aussi indiqué un retard dans l'installation de la saison des pluies d'au moins 20 jours dans quelques localités du Centre du Bénin. Ce résultat permet d'établir une relation intrinsèque entre le début 
de la saison des pluies et sa longueur. Cette relation a été mise en évidence dans les travaux de Guèye et Sivakumar (1992) au Sénégal. Houndenou et Hernandez (1998) ont abouti à la même conclusion au cours d'une étude semblable réalisée au Bénin. Ces différences, confirment les perturbations climatiques aux conséquences parfois dramatiques notamment sur 1'agriculture. Selon Adewi et al. (2010), les conséquences des changements de la saison des pluies utiles (début, fin et longueur) sur la durée et la position du cycle de végétation s'avèrent aussi contraignantes que la baisse du total pluviométrique. Il faudrait donc tenir compte de l'installation progressive de la saison des pluies dans la zone cotonnière pour semer au moment propice en sachant que chaque saison des pluies a ses propres caractéristiques. En effet, selon Ndong (2003), les semis précoces peuvent souffrir de séquences sèches à la levée comme aussi pendant la phase critique (floraison). Généralement cette phase intervient pendant la période la plus humide de la petite saison des pluies utiles. Par contre, le démarrage tardif de la saison des pluies peut porter préjudice aux variétés à cycle long, qui ne pourront pas boucler convenablement leur cycle, si la fin de la saison des pluies est plus précoce. Le choix de la date de semis doit donc être fait en dehors des risques. Il a été, en effet noté, qu'au cours de ces dernières années, les débuts et fins des saisons pluvieuses sont devenus de moins en moins prévisibles pour les exploitants agricoles (Sarr et al., 2011). Ainsi le stress hydrique, le risque de refaire le semis, le coût élevé de production, sont entre autres, autant de conséquences que peut entraîner cette perturbation de la saison culturale sur la productivité du coton en Côte d'Ivoire.

\section{Conclusion}

Cette étude a permis d'analyser la variabilité pluviométrique dans la zone cotonnière ivoirienne. Les indicateurs de la saison des pluies utiles ou saison culturale montrent une évolution spatio-temporelle contrastée dans la zone étudiée. En effet les hauteurs pluviométriques traduisent une tendance générale à la baisse et sont ponctuées par des périodes excédentaires, et déficitaires entre 1950 et 2000 . De façon générale, la fin des années 1960 et le début des années 1970 marquent la période de la baisse pluviométrique. Les paramètres de la saison de pluies utiles évoluent en fonction de la latitude. Les dates de début de la saison des pluies utiles sont de plus en plus tardives en montant en latitude et les longueurs des saisons des pluies sont de plus en plus courtes. La comparaison avant et après la rupture, des cumuls pluviométriques saisonniers montre des déficits allant jusqu'à $60 \%$. Toutefois, ces cumuls restent dans l'ensemble, potentiellement aptes à satisfaire les exigences hydriques du cotonnier. Les dates de début et de fin de la saison des pluies utiles sont devenues respectivement plus tardives et précoces après la rupture et les longueurs quant à elles plus courtes. Cette variabilité interannuelle des 
dates de démarrage et de fin de la saison des pluies utiles, pose des problèmes pour établir un calendrier cultural. Cette situation pourrait contribuer ainsi à la baisse la productivité du coton. Au regard de ces nouvelles conditions climatiques, l'actualisation du calendrier cultural s'impose.

\section{References:}

1. Adewi, E., Badameli, K., \& Dubreuil, V. (2010). Évolution des saisons des pluies potentiellement utiles au Togo de 1950 à 2000. Climatologie, 7 : 89-107.

2. Agbangla, A.D. (1998). Contribution à l'étude agroclimatique des saisons pluvieuses en vue de l'adaptation à la culture de l'arachide dans la province du Zou (Bénin). Mémoire d'ingénieur, Centre Régional AGHYMET, Niamey, 142p.

3. Ahoussi, K. E., Koffi Y. B., Kouassi, A. M., Soro, G., Soro N., Biémi J. (2013). Étude de la variabilité hydroclimatique et de ses conséquences sur les ressources en eau du Sud forestier et agricole de la Côte d'Ivoire : cas de la région d'Abidjan Agboville. International Journal of Pure \& Applied Bioscience. 1(6) : 30-50.

4. Assani, A. A. (1999). Analyse de la variabilité temporelle des précipitations (1916-1996) à Lumbashi (Congo-Kinshasa) en relation avec certains indicateurs de la circulation atmosphérique (oscillation australe) et océanique (El Niňo/La Niňa), Science et changements planétaires/Sécheresse.10(4) : 245-252.

5. Balme, M., Galle, S., \& Lebel, T. (2005). Démarrage de la saison des pluies au Sahel: variabilité aux échelles hydrologique et agronomique, analysée à partir des données EPSAT-Niger. Science et changements planétaires/Sécheresse, 16 (1): 15-22.

6. Boyer, J.F., Dieulin, C., Rouche N., Cres A., Servat E., Paturel J.E., Mahé G. (2006). SIEREM an environnemental information system for water resources. IAHS publication, vol. 308, p. 19

7. Cretenet, M., Follin, J.C., Hau, B., Letheve, C. H., Jallas, E. et Renou, A. (2002). Les plantes textiles. Dans Memento de l'agronome. CIRAD. Editions Quae Montpellier, pp. 1157-1198.

8. Diomandé, M. (2013). Impact du changement de pluviosité sur les systèmes de production agricoles en zone de contact forêt-savane de Côte d'Ivoire. Thèse unique, Université Félix Houphouët Boigny d'Abidjan (Côte d'Ivoire), 162p.

9. Dongo, K., Diomandé, M., Cissé, G., Tanner, M. et Biémi, J. (2007). Improving urban drainage in Abidjan Côte d'Ivoire. African Journal of Science and Technology. 8(1): 8-16.

10. Fossou, R. M. N., Lasm, T., Soro, N., Soro, T., Soro, G., De Lasme, O.Z., Baka, D., Onetie, O.Z. et Orou, R. (2015). Variabilité climatique 
et son impact sur les ressources en eaux souterraines: cas des stations de Bocanda et de Dimbokro au centre est de la Côte d'Ivoire (Afrique de 1'ouest). Larhyss Journal. 21 : 97-120

11. Goula, B. T. A., Srohourou, B., Brida, A., N’zué, K. A., \& Goroza, G. (2010). Determination and variability of growing seasons in Côte d'Ivoire. Int J Eng Sci, 2(11) : 5993-6003.

12. Goula, B. T. A., Kouassi, V. J. et Savane, I. (2006). Impacts du changement climatique sur les ressources en eau en zone tropicale humide: Cas du bassin-versant du bandama en Côte d'Ivoire. Agronomie Africaine. 18(1) :1-11.

13. Guèye, B. et Sivakumar, M.V.K. (1992). Analyse de la longueur de la saison culturale en fonction de la date de début des pluies au Sénégal. Compte rendu des travaux $\mathrm{n}^{\mathrm{o}} 2$. Niamey (Niger) : Centre sahélien de l'ICRISAT

14. Houndenou, C. et Hernandez, K. (1998). Modification de la saison pluvieuse dans l'Atakora (1961-1990). Un exemple de sécheresse au Nord-Ouest du Bénin (Afrique occidentale), Science et changements planétaires/Sécheresse, 9(1) :23-34.

15. Hubert, P., Carbonel, J. P., et Chaouche, A. (1989). Segmentation des séries hydrométéorologiques. Application à des séries de précipitations et de débits de l'Afrique de l'ouest. Journal of Hydrology, 110 : 349367

16. Koné, B. (2007). Utilisation des données pour 1'étude de la fertilité potentielle des sols ferralitiques au-dessus de la latitude $7^{\circ} \mathrm{N}$ de la Côte d'Ivoire. Thèse unique, Université de Cocody, 146p.

17. Lee A. F. S. et Heghinian S. M. (1977). A shift of the mean level in a sequence of independant normal random variables. A Bayesian approach. Technometrics 19: 503-506.

18. Mahé, G. et Olivry J.C. (1995). Variations des précipitations et des écoulements en Afrique de l'Ouest et centrale de 1951 à 1989. Science et changements planétaires/Sécheresse, 6(1) : 109-117.

19. Ndong, J.B. (2003). Caractérisation de la saison des pluies dans le Centre Ouest du Sénégal. Publication de l'Association Internationale de Climatologie, 15 : 326-332.

20. Nicholson, S.E., Kim, J., Hoopingarner, J. (1988). Atlas of African rainfall and its interannual variability. Edit. Department of Meteorology. Florida State University Tallahassee, Floride, USA, $237 \mathrm{p}$.

21. Noufé D. (2011) Changement hydroclimatique et transformation de l'agriculture : exemple des paysanneries de l'Est de la Côte d'Ivoire, Thèse de doctorat de géographie, hydrosciences Montpellier, Université Paris 1 Panthéon-Sorbonne, Paris, 375p. 
22. Noufé, D., Lidon, B., Mahé, G., Servat, E. et Chaléard, J. L. (2015). Impact de l'évolution des conditions agroclimatiques sur les systèmes de culture à base de banane plantain : le cas de l'Est ivoirien. VertigO la revue électronique en sciences de l'environnement, 15(1): 1-15. http://id.erudit.org/iderudit/1035750ar

23. Paturel, J. E., Servat, E., Kouamé, B. Et Boyer J. F. (1995). Manifestation de la sécheresse en Afrique de l'Ouest non sahélienne. Cas de la Côte d'Ivoire, du Togo et du Bénin. Science et changements planétaires/Sécheresse. 6(1): 95-102.

24. Paturel, J.E., Servat, E., Kouamé, B., Lubes, H., Masson, J.M., Boyer, J. F., Travaglio, M. et Marieu, B. (1997). Variabilité pluviométrique en Afrique humide le long du Golfe de Guinée. Approche régionale intégrée. Documents Techniques en Hydrologie, UNESCO, Paris, $16: 1-31$.

25. Pettitt, A. N. (1979). A non-parametric approach to the change-point problem. Applied statistics, $28: 126-135$. DOI : 10.2307/2346729

26. Revue des sciences de l'eau / Journal of Water Science. 26(3) : 247-26

27. Sarr B. (2007). Manuel d'utilisation destiné aux ingénieurs en agrométéorologie. Centre Régional AGHYMET, Niamey, 72p.

28. Sarr, B., Kafando, L. et Atta, S. (2011). Identification des risques climatiques de la culture du maïs au Burkina Faso. International Journal of Biological and Chemical Sciences, 5(4): 1659-1675. DOI: http://dx.doi.org/10.4314/ijbcs.v5i4.28

29. Servat, E., Paturel, J.E., Lubes-Niel, H., Kouamé, B., Masson, J.M., Travaglio, M. et Marieu B. (1999). De différents aspects de la variabilité de la pluviométrie en Afrique de l'ouest et centrale non sahélienne. Revue des sciences de l'eau/Journal of Water Science.12(2) :363-387.

30. Sivakumar M. (1988). Predicting rainy season potential from the onset of rains in the southern sahelian and soudanian climatic zone of West Africa. Agricultural and forest meteorology, 42: 295-305.

31. Stern, R., Rijks, D., Dale I., Knock, J. (2006). instat Climatic Guide INSTAT+ for Windows V3.036 Statistical Services Center, University of Reading: Reading. 330p. 